
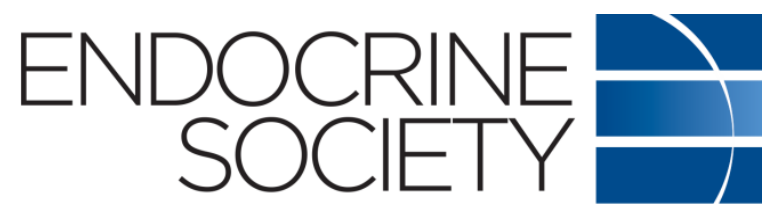

\title{
Associations of outdoor temperature, bright sunlight and cardiometabolic traits in two European population-based cohorts
}

\author{
Raymond Noordam, Ashna Ramkisoensing, Nellie Y Loh, Matt J Neville, Frits R \\ Rosendaal, Ko Willems van Dijk, Diana van Heemst, Fredrik Karpe, Constantinos \\ Christodoulides, Sander Kooijman
}

\section{The Journal of Clinical Endocrinology \& Metabolism Endocrine Society}

Submitted: November 24, 2018

Accepted: February 07, 2019

First Online: February 12, 2019

Advance Articles are PDF versions of manuscripts that have been peer reviewed and accepted but not yet copyedited. The manuscripts are published online as soon as possible after acceptance and before the copyedited, typeset articles are published. They are posted "as is" (i.e., as submitted by the authors at the modification stage), and do not reflect editorial changes. No corrections/changes to the PDF manuscripts are accepted. Accordingly, there likely will be differences between the Advance Article manuscripts and the final, typeset articles. The manuscripts remain listed on the Advance Article page until the final, typeset articles are posted. At that point, the manuscripts are removed from the Advance Article page.

DISCLAIMER: These manuscripts are provided "as is" without warranty of any kind, either express or particular purpose, or non-infringement. Changes will be made to these manuscripts before publication. Review and/or use or reliance on these materials is at the discretion and risk of the reader/user. In no event shall the Endocrine Society be liable for damages of any kind arising references to, products or publications do not imply endorsement of that product or publication. 
"Temperature, sunlight and cardiometabolic traits"

\title{
Associations of outdoor temperature, bright sunlight and cardiometabolic traits in two European population-based cohorts
}

\author{
Raymond Noordam ${ }^{1}$, Ashna Ramkisoensing ${ }^{2}$, Nellie Y Loh ${ }^{3}$, Matt J Neville ${ }^{3,4}$, Frits R
} Rosendaal $^{5}$, Ko Willems van Dijk ${ }^{6,7}$, Diana van Heemst ${ }^{1}$, Fredrik Karpe ${ }^{3,4}$, Constantinos Christodoulides $^{3, \$}$, Sander Kooijman ${ }^{3,7}$

\begin{abstract}
${ }^{1}$ Department of Internal Medicine, Section of Gerontology and Geriatrics, Leiden University Medical Center, Leiden, the Netherlands. ${ }^{2}$ Department of Molecular Cell Biology, Division of Neurophysiology, Leiden University Medical Center, Leiden, the Netherlands. ${ }^{3}$ Oxford Centre for Diabetes, Endocrinology and Metabolism, Radcliffe Department of Medicine, University of Oxford, Oxford, United Kingdom. ${ }^{4}$ NIHR Oxford Biomedical Research Centre, OUH Foundation Trust, Oxford, UK. ${ }^{5}$ Department of Clinical Epidemiology, Leiden University Medical Center, Leiden, the Netherlands. ${ }^{6}$ Department of Human Genetics, Leiden University Medical Center, Leiden, the Netherlands. ${ }^{7}$ Department of Internal Medicine, Division of Endocrinology, Leiden University Medical Center, Leiden, the Netherlands.
\end{abstract}

ORCiD numbers:

0000-0001-7801-809X

Noordam

Raymond

Received 24 November 2018. Accepted 07 February 2019.

Context: Seasonal variation in cold and light exposure may influence metabolic health. Objective: We assessed the associations of bright sunlight and outdoor temperature with measures of glucose and lipid metabolism in two populations of middle-aged European subjects.

Design: Cross-sectional study.

Setting: Two population-based European cohorts.

Patients or Other Participants: Middle-aged non-diabetic subjects from the Oxford Biobank (OBB; $\mathrm{N}=4,327$; mean age 41.4 years) and the Netherlands Epidemiology of Obesity Study (NEO; $\mathrm{N}=5,899$; mean age 55.6 years).

Intervention(s): Data on outdoor bright sunlight and temperature collected from local weather stations.

Main Outcome Measure(s): Insulin resistance and fasting lipid levels.

Statistical analyses: Multivariable regression analyses adjusted for age, sex, percentage body fat, season, and either outdoor temperature or bright sunlight.

Results: In the OBB cohort, increased bright sunlight exposure was associated with lower fasting insulin $(-1.27 \%$ [95\% confidence interval: $-2.09,-0.47]$ per extra hour of bright sunlight), lower homeostatic model assessment for insulin resistance $(-1.36 \%[-2.23,-0.50])$, lower homeostatic model assessment for beta cell function $(-0.80 \%[-1.31,-0.30])$, and lower triglyceride $(-1.28 \%$ [-2.07, -0.50$])$ levels. In the NEO cohort generally unidirectional but weaker associations were observed. No associations between outdoor temperature and measures of glucose or lipid metabolism were detected following adjustment for bright sunlight.

Conclusions: Bright sunlight, but not outdoor temperature, might be associated with increased insulin sensitivity and lower triglyceride levels.

Outdoor temperature and sunlight were studied in relation to cardiometabolic traits and it was found that specifically outdoor sunlight showed beneficial associations on insulin resistance. . 


\section{Introduction}

Several studies have been conducted to date to identify modifiable (e.g., lifestyle) and nonmodifiable (e.g., genetic variation) risk factors for developing cardiometabolic disease. Recent preclinical and clinical studies have linked environmental temperature to cardiometabolic health, with thermogenesis in muscle and brown adipose tissue as potential mediators (1-7). For example, Blauw et al. (7) reported an increased incidence of type 2 diabetes (T2D) with higher mean annual outdoor temperature both across US states and worldwide. Similarly, Speakman \& Heidari-Bakavoli (8) calculated that ambient temperature explained $12.4 \%$ of the variation in the prevalence of T2D across mainland USA after accounting for obesity, poverty and race. These findings may be attributable to lifestyle adaptations to changes in outdoor temperature, but it has also been hypothesized that metabolism can rapidly adapt to changes in environmental temperature. For example, seasonal fluctuations in measures of insulin sensitivity were reported in the Rotterdam study cohort, which could only partly be attributed to seasonal changes in lifestyle $(9,10)$. Furthermore, using a hyperinsulinemic euglycemic clamp, researchers in another study (11) found that insulin sensitivity was lowest during winter in addition to demonstrating a positive effect of outdoor temperature on insulin sensitivity irrespective of season. More directly, Hanssen et al showed that short-term cold acclimation improves insulin sensitivity in patients with T2D, an effect which was associated with markedly increased basal skeletal muscle GLUT4 translocation (5).

As outdoor temperature is associated with outdoor bright sunlight, the interpretation of these studies is complicated. Increased exposure to bright sunlight can increase nocturnal melatonin levels (12), which in turn inhibit pancreatic insulin secretion (13). Vitamin D which is primarily produced in the skin in response to sunlight exposure has also been linked with the development of insulin resistance (14). In addition, brown adipose tissue exhibits a daily rhythm in glucose and lipid uptake $(15,16)$, which at least in rodents, is modulated by the duration of daily light exposure (16). In this study, we aimed to examine the associations of outdoor temperature and bright sunlight with measures of body composition, insulin sensitivity and fasting lipid concentrations in a combined cohort of $>10,000$ middle-aged healthy European subjects enrolled in the Oxford Biobank (OBB) and the Netherlands Epidemiology of Obesity (NEO) study.

\section{Methods}

\section{Study settings}

The OBB is a population-based cohort of randomly selected healthy participants aged 30 to 50 years from Oxfordshire (UK). Individuals with a history of myocardial infarction, diabetes mellitus, heart failure, untreated malignancy, other ongoing systemic diseases or ongoing pregnancy were not eligible for study inclusion. Enrolment of participants started in 1999 and as of May 2015 the OBB cohort comprised 7,185 individuals (4,054 women and 3,131 men). A more detailed description of the study recruitment criteria and population characteristics is reported elsewhere (17).

The NEO study is population-based prospective cohort study of men and women aged between 45 and 65 years with an oversampling of individuals with a BMI of $27 \mathrm{~kg} / \mathrm{m}^{2}$ or higher, living in the greater area of Leiden (in the West of the Netherlands). In addition, all inhabitants aged between 45 and 65 years from one municipality (Leiderdorp) were invited in the study irrespective of their BMI, to allow for a reference distribution of BMI. Between September 2008 and September 2012, 6,671 individuals were included in the study. Detailed information about the study design and data collection has been described previously (18).

In both cohorts, participants were invited for a detailed baseline assessment, conducted after an overnight fast, which included blood sampling and anthropometry. Both studies were 
approved by local ethics committees, and written informed consent was obtained from all study participants.

Study population

In the OBB, we excluded individuals with missing data on mean outdoor temperature and bright sunlight on the day of the study visit $(\mathrm{N}=406)$ and individuals with missing data on body composition $(\mathrm{N}=2,452)$. From $\mathrm{NEO}$, we excluded individuals with both treated and diagnosed diabetes, as well as, subjects with a fasting glucose concentration above 7.0 $\mathrm{mmol} / \mathrm{L}(\mathrm{N}=749)$ in order to have a uniform population, in terms of glycaemic status, as that of the OBB. In addition, we excluded participants who were not fasted (self-reported; $N=23$ ).

Data collection on outdoor temperature and bright sunlight

Data on mean outdoor temperature and hours of bright sunlight (defined as global radiation $>120 \mathrm{~W} / \mathrm{m}^{2}$ ) were collected from local weather stations. Based on these data, we calculated the mean outdoor temperature and bright sunlight duration over a 7-and 30-day period before the date of blood sampling. For the OBB data were obtained from the Radcliffe Meteorological Station (School of Geography and the Environment, University of Oxford, UK) whilst for the NEO study we obtained meteorological data from the Koninklijk Nederlands Meteorologisch Instituut (Royal Dutch Meteorological Institute) which has the closest proximity to the city of Leiden.

Laboratory assays

In the OBB, fasting glucose, total cholesterol and triglyceride concentrations were measured in plasma using Instrumentation Laboratory IL Test ${ }^{\mathrm{TM}}$ kits on an ILab 600/650 clinical chemistry analyzers (Werfen, Warrington, UK). HDL- and LDL-cholesterol levels were measured in plasma using the Randox direct clearance method adapted for use on the ILab 600/650 analysers (Randox Laboratories, Crumlin, Northern Ireland). Fasting insulin levels were measured with the Millipore Human Insulin specific radioimmunoassay (Millipore UK, Watford, UK).

In NEO, fasting serum glucose, total cholesterol and triglycerides concentrations were determined by enzymatic colorimetric methods (Roche Modular Analytics P800, Roche Diagnostics, Mannheim, Germany; CV < 5\%). HDL-cholesterol concentration was measured with the homogenous HDLc method ( $3{ }^{\text {rd }}$ generation; Roche Modular Analytics P800, Roche Diagnostics, Mannheim, Germany; CV < 5\%); Serum insulin concentration was measured with an immunometric method (Siemens Immulite 2500, Siemens Healthcare Diagnostics, Breda, The Netherlands; CV < 5\%). LDL-cholesterol concentration was estimated using the Friedewald formula (19)

In both cohorts, the Homeostatic Model Assessment for Insulin Resistance (HOMA-IR) was calculated as [fasting insulin $(\mu \mathrm{U} / \mathrm{mL})]^{*}[$ fasting glucose $(\mathrm{mmol} / \mathrm{L})] / 22.5$, and the Homeostatic Model Assessment for Insulin Secretion (HOMA-B) was calculated as $[20 \mathrm{x}$ fasting insulin $(\mu \mathrm{U} / \mathrm{mL})] /[$ glucose $(\mathrm{mmol} / \mathrm{L})-3.5](20,21)$.

Measurements

In the OBB, body fat percentage was determined by Dual-energy X-ray absorptiometry using a GE Lunar iDXA. All data were analyzed with Encore software (version 11.0; GE. Medical Systems, Madison, WI, USA). For NEO, body weight and percent body fat were measured by the Tanita bioimpedance balance (TBF-310, Tanita International Division, UK). Height and weight were measured by research nurses at the OBB and NEO study centers. BMI was calculated by dividing the weight in kilograms by the height in meters squared. Season was derived from the date of the blood sampling (winter: December - February, spring: March May: summer: June - August; autumn: September - November). Use of lipid-lowering medication was determined from the medication inventory. 
Statistical analyses

In the NEO study, individuals with a BMI of $27 \mathrm{~kg} / \mathrm{m}^{2}$ or higher were oversampled. To correctly represent associations for the general population (22), adjustments for the oversampling of participants with a BMI $\geq 27 \mathrm{~kg} / \mathrm{m}^{2}$ were made. This was done by weighting all participants towards the BMI distribution of participants from the Leiderdorp municipality (23), whose BMI distribution was similar to the BMI distribution of the general Dutch population (18). All results in NEO were based on weighted analyses. Consequently, the results apply to a population-based study without oversampling of individuals with a $B M I \geq 27$ $\mathrm{kg} / \mathrm{m}^{2}$. As a result of the weighting procedure, the numbers of participants per group in NEO are presented as percentages.

All analyses in the OBB and NEO were performed using STATA version 12.1 (StataCorp LP, TX, US). Characteristics of the OBB and NEO study populations separately were expressed as (weighted) mean (with standard deviation [SD], (weighted) median (inter quartile range [IQR]) or (weighted) proportion (\%).

As most of the outcome variables were not normally distributed, we log-transformed all outcome variables to be able to better compare the effect sizes of the different study outcomes. Associations of mean bright sunlight and temperature with measures of insulin resistance and dyslipidemia were examined using multivariable linear regression analyses separately for the OBB and NEO study populations. Estimates retrieved from the analyses in the OBB and NEO study populations were subsequently meta-analyzed using inversevariance weighting using the rmeta statistical package in $\mathrm{R}$. Beta estimates from the separate results and results from the meta-analyses were back-transformed and were expressed as the percentage difference in outcome variable per unit increase in outdoor temperature or bright sunlight $(95 \%$ confidence interval $(95 \% \mathrm{CI}))$. Model 1 was adjusted for age, sex and percentage body fat. Model 2 was additionally adjusted for season. Model 3 was additionally adjusted either for the mean temperature or mean hours of bright sunlight. In sensitivity analyses on blood lipid levels, we further adjusted for use of lipid-lowering medication. We additionally adjusted all associations for BMI rather than for percentage body fat.

Furthermore, we repeated the analyses using a mean temperature/bright sunlight exposure over 30 days as exposure. For presentation purposes, we analyzed the data per 5 degrees Celsius increase in temperature and per hour increase in sunlight exposure.

Results

\section{Characteristics of the study populations}

The total study population ( $\mathrm{N}=10,226)$ comprised of 4,327 individuals from the $\mathrm{OBB}$ and 5,899 individuals from the NEO study (Table 1). Participants from the OBB were younger (mean age 41.4 versus 55.6 years) and had higher mean percentage body fat (42.1 versus $31.5 \%$ ) compared with participants from the NEO study. Median insulin concentrations (11.5 versus $7.5 \mathrm{mIU} / \mathrm{L}$ ), median HOMA-IR values (2.6 versus 1.8) and median HOMA-B values (106 versus 87), were higher in the OBB than in the NEO cohort whilst use of lipid-lowering medication was higher in NEO (8.2 versus $0.7 \%)$. Mean outdoor temperature was similar in the two geographic regions, although the median number of hours of bright sunlight duration was lower in Oxfordshire than in the Leiden area during the study period (3.8 versus 5.0 hours).

Temperature and bright sunlight during a representative 3-months period for Oxfordshire and Leiden are presented in Supplementary Figure 1 (24). Whilst bright sunlight duration fluctuated markedly on a day-to-day basis variation variation in temperature was less pronounced. As expected, a higher number of hours of bright sunlight was associated with a higher outdoor temperature (1.30 degrees Celsius per 1 extra hour of bright sunlight [95\%CI: $1.25,1.35$ ] in Oxfordshire and 0.96 per 1 extra hour of bright sunlight [95\% CI: 0.91, 1.01] in 
Leiden). Independent of season, outdoor bright sunlight was associated with a lower BMI in the OBB cohort $\left(-0.09 \mathrm{~kg} / \mathrm{m}^{2}\right.$ per 1 hour [95\%CI: $\left.\left.-0.16,-0.02\right]\right)$ and a higher BMI in the NEO cohort $\left(0.06 \mathrm{~kg} / \mathrm{m}^{2}\right.$ per 1 hour [95\%CI: $\left.\left.0.00,0.12\right]\right)$. No association between duration of bright sunlight and percentage total body fat was observed in either cohort. Independent of season, a higher outdoor temperature was associated with a lower BMI in both the OBB cohort $(-0.19$ $\mathrm{kg} / \mathrm{m}^{2}$ per 5 degrees Celsius [95\%CI: $\left.\left.-0.43,0.05\right]\right)$ and the NEO cohort $\left(-0.44 \mathrm{~kg} / \mathrm{m}^{2}\right.$ per 5 degrees Celsius [95\%CI: $-0.62,-0.25]$ ). Directionally similar associations were observed for percentage total body fat in both populations.

\section{Outdoor temperature and glucose and lipid metabolism}

Cohort-specific results for associations between outdoor temperature and measures of glucose and lipid metabolism are presented in Table 2. After adjustment for age, sex, and percentage body fat, higher mean outdoor temperature during the 7 days prior to the center visit was associated with lower HOMA-IR (-1.42\% per 5 degrees Celsius increase in mean outdoor temperature [95\% CI: $-2.86,-0.01])$ in the OBB. This association became stronger after additional adjustment for season $(-3.11 \%$ [95\%CI: $-5.74,-0.54])$ and was primarily driven by lower glucose levels at higher mean outdoor temperatures $(-0.51 \%$ [95\% CI -0.92, -0.09]). However, both associations attenuated towards the null after additional adjustment for mean bright sunlight. In the NEO cohort, no associations between mean outdoor temperature and glucose or lipid metabolism were detected in any of the models studied.

In the meta-analysis, only the association between mean outdoor temperature and HOMAIR persisted after adjustment for age, sex, percentage body fat and season (-2.15\% [95\%CI: 4.16, -0.18]; Supplementary Table 1) (24). Additional adjustment for mean daily hours of outdoor bright sunlight attenuated this association (-1.04\% [95\%CI: $-3.22,1.10])$. Results were not materially different when we adjusted associations for BMI rather than percentage body fat. When taking into account the mean outdoor temperature during the 30 days prior to the center visit in the basic model (Supplementary Table 2) (24), the association with HOMA-IR became stronger (-1.22\% [95\%CI: -2.44, -0.01]). No associations between mean outdoor temperature and glucose or lipid levels were detected although, as highlighted in the preceding paragraph, we observed heterogeneity between the two cohorts.

\section{Bright sunlight and glucose and lipid metabolism}

Cohort-specific results on the associations between bright sunlight hours and measures of glucose and lipid metabolism are presented in Table 3. In the OBB, after adjustment for age, sex, and percentage body fat, longer mean bright sunlight duration was associated with lower HOMA-IR (-1.05\% [95\%CI: -1.65, -0.45]) and lower HOMA-B (-0.54\% [95\%CI: -0.89, $0.19])$. Both these associations were driven by lower fasting insulin levels $(-0.92 \%$ per hour increase in bright sunlight [95\%CI: -1.48, -0.36]) in the presence of prolonged sunshine. Longer mean bright sunlight hours were additionally associated with lower LDL-cholesterol ($0.36 \%$ [95\% CI: $-0.66,-0.07])$, and lower triglyceride levels $(-0.57 \%$ [95\%CI: $-1.12,-0.03])$. With the exception of LDL-cholesterol, all associations persisted and indeed became stronger after adjustment for season and mean outdoor temperature. No association between outdoor bright sunlight hours and glucose concentration was detected. In the NEO cohort, associations between bright sunlight hours and glucose and lipid traits were in the same direction as those observed in the OBB but with smaller effect sizes.

In the meta-analysis (Supplementary Table 3) results did not materially differ from the data presented for the OBB cohort (24). This was also true after we adjusted our data for BMI rather than percentage body fat (data not shown) or when we additionally adjusted the associations between bright sunlight duration and lipid levels for use of lipid-lowering medication. Specifically after meta-analyses, the results become somewhat weaker considering a 30-days period to calculate the mean hours of bright sunlight (Supplementary Table 4) (24). 


\section{Discussion}

In the present study, we examined the associations of outdoor temperature and bright sunlight with measures of glucose and lipid metabolism. Using two independent study populations with a combined sample size of more than 10,000 non-diabetic subjects of European descent, we detected little evidence for associations between mean outdoor temperature and glucose or lipid traits. In contrast, we found that bright sunlight was associated with a 'healthier' metabolic profile conditional on season and mean outdoor temperature. These associations between bright sunlight duration and measures of glucose and lipid metabolism were generally stronger in the OBB than in NEO. This may have been due to differences in body composition and/or metabolic profile between the two cohorts. Nevertheless, our study is the first to suggest that regular exposure to bright sunlight could improve glucose and lipid metabolism, thereby decreasing the lifetime risk of developing cardiometabolic disease.

Previous studies have suggested a link between lower outdoor temperature and reduced T2D risk with thermogenesis in muscle and brown adipose tissue as potential mediators $(7,8)$. When taking into account the mean outdoor temperature in the 30 days prior to the center visit we found that, in non-diabetic subjects, a higher mean outdoor temperature was associated with lower HOMA-IR. We suspect that this might be primarily related to increased physical activity and/or altered habitual food intake when the outdoor temperature starts to increase as evidenced by the association of higher outdoor temperature with lower BMI in both cohorts, although we were not able to test this hypothesis. At least from the perspective in which cold acclimatization promotes the thermogenic activity of brown adipose tissue (25-27), this was an unexpected result. We can think of several reasons for the lack of expected associations. First, our study populations comprised primarily of middle-aged overweight subjects. Both age and BMI are known to be inversely associated with the amount and activity of brown adipose tissue as measured by the uptake of $\left[{ }^{18} \mathrm{~F}\right]$ fluorodeoxyglucose (25). We note, however, that older and heavier subjects may benefit most from strategies that promote brown fat activation and are therefore an important population to study. Secondly, the impact of mean outdoor temperature on cardiometabolic outcomes could have been diluted due to controlled in-door climate and clothing habits. To further dissect the roles of outdoor temperature and bright sunlight on systemic metabolism whilst simultaneously accounting for physical activity larger studies with more dense phenotyping are required.

To the best of our knowledge, this is the first human population study to show a positive association between hours of ambient bright sunlight and metabolic health. We acknowledge that we have no data available as to whether the participating individuals in our study were actually exposed to more sunlight during days with more sunshine. Nonetheless, such effects would dilute the exposure variables and hence the true effect of bright sunlight on measures of glucose and lipid metabolism may actually be larger than observed here. One candidate mechanism via which bright sunlight exposure may improve insulin sensitivity and lipid metabolism is through enhanced vitamin D generation (14). However, this is unlikely due to the lack of a causal association between vitamin D and T2D (28). A more conceivable biological mechanism, in addition to light-associated changes in diet and/or lifestyle, is the involvement of melatonin signaling; with nocturnal melatonin concentrations increasing with prolonged exposure to bright light (12). In turn, increased nocturnal melatonin levels may lead to reduced insulin secretion, as has been demonstrated in rodent studies (13). Interestingly, a role for melatonin in glucose metabolism has also been demonstrated by genetic association studies; common genetic variation in $M T N R 1 B$, which encodes one of the melatonin receptors, was associated with fasting glucose levels and T2D risk (29) and rare partial- and total loss-of-function MTNR1B variants were associated with increased T2D risk (30). Furthermore, clinical trials have revealed that treatment with melatonin improves blood pressure and lipid metabolism in subjects with the metabolic syndrome (31). Based on these 
findings and our own data, we postulate that bright sunlight exposure might be of benefit to individuals with an unhealthy cardiometabolic risk profile through activation of melatonin signaling. Indeed, an ongoing clinical trial is currently assessing the role of light therapy in improving insulin sensitivity in patients with depression and T2D (32). Further results from such studies are necessary to determine the clinical relevance of our findings and to test our hypothesis that altered melatonin signaling mediates the beneficial effects of bright sunlight exposure on systemic metabolism.

The present study has a number of strengths and limitations. The study made use of data collected from 2 independent cohorts with a combined sample size of more than 10,000 participants. Another strength of our study was that both the OBB and NEO participants were residing in relatively small geographic areas, hence we were able to accurately couple data from local weather stations with anthropometric and biochemical data collected during the center visits. Limitations include that, effect sizes were small, and whilst generally unidirectional in the two study populations, associations were generally stronger in OBB than in NEO. This is likely due to differences in population characteristics as discussed above (see also Table 1). Due to this inherent heterogeneity in cohort characteristics, the results of the meta-analyses should be interpreted with caution. Given that all outcome variables, especially those related to glucose metabolism, are highly interrelated we did not correct our data for multiple testing. Notwithstanding, associations between bright sunlight duration and metabolic measures within the OBB were robust and most would have survived multiple testing correction. Finally, we were unable to examine the potential contribution of lifestyle factors such as habitual food intake, sleep patterns, and physical activity to the observed associations. However, none of these factors can affect our exposures i.e. outdoor temperature and bright sunlight duration. Hence, instead of acting as confounders these factors are more likely to act as mediators and could potentially explain the biological mechanisms behind our observations.

In summary, in this large study we have found evidence of an association between bright sunlight, but not outdoor temperature, and a healthier metabolic profile. Although all associations had a similar direction of effect in both of our study cohorts, positive findings were predominantly observed in the OBB population, and only to a lesser extent in the NEO cohort. In addition, causality as well as the potential direct effects of sunlight on a healthier metabolic profile remain to be investigated.

\section{Acknowledgements}

We express our gratitude to all individuals who participate in the OBB and NEO study. We are grateful to all participating general practitioners for inviting eligible participants and all research nurses for collecting the data. The Oxford Biobank is supported by the NIHR Oxford BRC Obesity and Lifestyle theme. The NEO study is supported by the participating Departments, the Division and the Board of Directors of the Leiden University Medical Center, and by the Leiden University, Research Profile Area 'Vascular and Regenerative Medicine'.

\section{Funding}

FK is supported by a British Heart Foundation (BHF) programme grant (RG/17/1/32663). CC is a BHF Intermediate Clinical Research Fellow. DvH is supported by the European Commission funded project HUMAN (Health-2013-INNOVATION-1-602757). SK is supported by the Dutch Heart Foundation (2017T016) and the European Foundation for the Study of Diabetes (EFSD Albert Renold Travel Fellowship and EFSD Rising Star Fellowship Programme). We acknowledge the support of the Netherlands Cardiovascular Research Initiative: an initiative with the support of the Dutch Heart Foundation (CVON2014-02 ENERGISE). 
FP7 Health http://dx.doi.org/10.13039/100011272, Health-2013-INNOVATION-1602757, Diana van Heemst; Hartstichting http://dx.doi.org/10.13039/501100002996, CVON2014-02 ENERGISE, Frits R Rosendaal; European Foundation for the Study of Diabetes http://dx.doi.org/10.13039/501100001648, Renold Travel Fellowship, Sander Kooijman; British Heart Foundation http://dx.doi.org/10.13039/501100000274, RG/17/1/32663, Fredrik Karpe; European Foundation for the Study of Diabetes http://dx.doi.org/10.13039/501100001648, Rising Star Fellowship, Sander Kooijman; British Heart Foundation http://dx.doi.org/10.13039/501100000274, Clinical Research Fellow, Constantinos Christodoulides; Hartstichting http://dx.doi.org/10.13039/501100002996, 2017T016, Sander Kooijman

\$Corresponding author: Constantinos Christodoulides, Radcliffe Department of Medicine, Oxford Centre for Diabetes, Endocrinology and Metabolism, University of Oxford, OX3 7LE, Oxford, United Kingdom; E-mail:

Costas.Christodoulides@ocdem.ox.ac.uk

\section{Conflict of interest}

The authors declare that there is no conflict of interest regarding the publication of this article.

\section{References}

1. Bartelt A, John C, Schaltenberg N, Berbee JFP, Worthmann A, Cherradi ML, Schlein C, Piepenburg J, Boon MR, Rinninger F, Heine M, Toedter K, Niemeier A, Nilsson SK, Fischer M, Wijers SL, van Marken Lichtenbelt W, Scheja L, Rensen PCN, Heeren J. Thermogenic adipocytes promote HDL turnover and reverse cholesterol transport. Nature communications. 2017;8:15010.

2. Berbee JF, Boon MR, Khedoe PP, Bartelt A, Schlein C, Worthmann A, Kooijman S, Hoeke G, Mol IM, John C, Jung C, Vazirpanah N, Brouwers LP, Gordts PL, Esko JD, Hiemstra PS, Havekes LM, Scheja L, Heeren J, Rensen PC. Brown fat activation reduces hypercholesterolaemia and protects from atherosclerosis development. Nature communications. 2015;6:6356.

3. Bartelt A, Bruns OT, Reimer R, Hohenberg H, Ittrich H, Peldschus K, Kaul MG, Tromsdorf UI, Weller H, Waurisch C, Eychmuller A, Gordts PL, Rinninger F, Bruegelmann K, Freund B, Nielsen P, Merkel M, Heeren J. Brown adipose tissue activity controls triglyceride clearance. Nature medicine. 2011;17(2):200-205.

4. Bakker LE, Boon MR, van der Linden RA, Arias-Bouda LP, van Klinken JB, Smit F, Verberne HJ, Jukema JW, Tamsma JT, Havekes LM, van Marken Lichtenbelt WD, Jazet IM, Rensen PC. Brown adipose tissue volume in healthy lean south Asian adults compared with white Caucasians: a prospective, case-controlled observational study. The lancet Diabetes \& endocrinology. 2014;2(3):210-217.

5. Hanssen MJ, Hoeks J, Brans B, van der Lans AA, Schaart G, van den Driessche JJ, Jorgensen JA, Boekschoten MV, Hesselink MK, Havekes B, Kersten S, Mottaghy FM, van Marken Lichtenbelt WD, Schrauwen P. Short-term cold acclimation improves insulin sensitivity in patients with type 2 diabetes mellitus. Nature medicine. 2015;21(8):863-865.

6. van der Lans AA, Hoeks J, Brans B, Vijgen GH, Visser MG, Vosselman MJ, Hansen J, Jorgensen JA, Wu J, Mottaghy FM, Schrauwen P, van Marken Lichtenbelt WD. Cold acclimation recruits human brown fat and increases nonshivering thermogenesis. The Journal of clinical investigation. 2013;123(8):3395-3403.

7. Blauw LL, Aziz NA, Tannemaat MR, Blauw CA, de Craen AJ, Pijl H, Rensen PC. Diabetes incidence and glucose intolerance prevalence increase with higher outdoor temperature. BMJ open diabetes research \& care. 2017;5(1): $\mathrm{e} 000317$. 
8. Speakman JR, Heidari-Bakavoli S. Type 2 diabetes, but not obesity, prevalence is positively associated with ambient temperature. Sci Rep. 2016;6:30409.

9. Cepeda M, Koolhaas CM, Van Rooij FJ, Tiemeier H, Guxens M, Franco OH, Schoufour JD. Seasonality of physical activity, sedentary behavior, and sleep in a middle-aged and elderly population: The Rotterdam study. Maturitas. 2018;110:41-50.

10. Cepeda M, Muka T, Ikram MA, Franco OH, Schoufour JD. Seasonality of Insulin Resistance, Glucose, and Insulin Among Middle-Aged and Elderly Population: The Rotterdam Study. The Journal of clinical endocrinology and metabolism. 2018;103(3):946955.

11. Berglund L, Berne C, Svardsudd K, Garmo H, Melhus H, Zethelius B. Seasonal variations of insulin sensitivity from a euglycemic insulin clamp in elderly men. Ups J Med Sci. 2012;117(1):35-40.

12. Park SJ, Tokura H. Bright light exposure during the daytime affects circadian rhythms of urinary melatonin and salivary immunoglobulin A. Chronobiol Int. 1999;16(3):359-371.

13. Peschke E, Bahr I, Muhlbauer E. Melatonin and pancreatic islets: interrelationships between melatonin, insulin and glucagon. Int J Mol Sci. 2013;14(4):6981-7015.

14. Pilz S, Kienreich K, Rutters F, de Jongh R, van Ballegooijen AJ, Grubler M, Tomaschitz A, Dekker JM. Role of vitamin D in the development of insulin resistance and type 2 diabetes. Current diabetes reports. 2013;13(2):261-270.

15. Lee P, Bova R, Schofield L, Bryant W, Dieckmann W, Slattery A, Govendir MA, Emmett L, Greenfield JR. Brown Adipose Tissue Exhibits a Glucose-Responsive Thermogenic Biorhythm in Humans. Cell metabolism. 2016;23(4):602-609.

16. van den Berg R, Kooijman S, Noordam R, Ramkisoensing A, Abreu-Vieira G, Tambyrajah LL, Dijk W, Ruppert P, Mol IM, Kramar B, Caputo R, Puig LS, de Ruiter EM, Kroon J, Hoekstra M, van der Sluis RJ, Meijer OC, Willems van Dijk K, van Kerkhof LWM, Christodoulides C, Karpe F, Gerhart-Hines Z, Kersten S, Meijer JH, Coomans CP, van Heemst D, Biermasz NR, Rensen PCN. A Diurnal Rhythm in Brown Adipose Tissue Causes Rapid Clearance and Combustion of Plasma Lipids at Wakening. Cell reports. 2018;22(13):3521-3533.

17. Karpe F, Vasan SK, Humphreys SM, Miller J, Cheeseman J, Louise Dennis A, Neville MJ. Cohort Profile: The Oxford Biobank. International journal of epidemiology. 2017.

18. de Mutsert R, den Heijer M, Rabelink TJ, Smit JW, Romijn JA, Jukema JW, de Roos A, Cobbaert CM, Kloppenburg M, le Cessie S, Middeldorp S, Rosendaal FR. The Netherlands Epidemiology of Obesity (NEO) study: study design and data collection. European journal of epidemiology. 2013;28(6):513-523.

19. Friedewald WT, Levy RI, Fredrickson DS. Estimation of the concentration of lowdensity lipoprotein cholesterol in plasma, without use of the preparative ultracentrifuge. Clin Chem. 1972;18(6):499-502.

20. Matthews DR, Hosker JP, Rudenski AS, Naylor BA, Treacher DF, Turner RC. Homeostasis model assessment: insulin resistance and beta-cell function from fasting plasma glucose and insulin concentrations in man. Diabetologia. 1985;28(7):412-419.

21. Wallace TM, Levy JC, Matthews DR. Use and abuse of HOMA modeling. Diabetes Care. 2004;27(6):1487-1495.

22. Korn EL, Graubard BI. Epidemiologic studies utilizing surveys: accounting for the sampling design. Am J Public Health. 1991;81(9):1166-1173.

23. Lumley T. Analysis of complex survey samples. 2004.

24. Supplementary Tables, available at https://figshare.com/s/af69f38a950879d2709c (last assessed January 26, 2019). 
25. van Marken Lichtenbelt WD, Vanhommerig JW, Smulders NM, Drossaerts JM, Kemerink GJ, Bouvy ND, Schrauwen P, Teule GJ. Cold-activated brown adipose tissue in healthy men. The New England journal of medicine. 2009;360(15):1500-1508.

26. Chen KY, Brychta RJ, Linderman JD, Smith S, Courville A, Dieckmann W, Herscovitch P, Millo CM, Remaley A, Lee P, Celi FS. Brown fat activation mediates coldinduced thermogenesis in adult humans in response to a mild decrease in ambient temperature. The Journal of clinical endocrinology and metabolism. 2013;98(7):E1218-1223. 27. Au-Yong IT, Thorn N, Ganatra R, Perkins AC, Symonds ME. Brown adipose tissue and seasonal variation in humans. Diabetes. 2009;58(11):2583-2587.

28. Ye Z, Sharp SJ, Burgess S, Scott RA, Imamura F, InterAct C, Langenberg C, Wareham NJ, Forouhi NG. Association between circulating 25-hydroxyvitamin D and incident type 2 diabetes: a mendelian randomisation study. The lancet Diabetes \& endocrinology. 2015;3(1):35-42.

29. Bouatia-Naji N, Bonnefond A, Cavalcanti-Proenca C, Sparso T, Holmkvist J, Marchand M, Delplanque J, Lobbens S, Rocheleau G, Durand E, De Graeve F, Chevre JC, Borch-Johnsen K, Hartikainen AL, Ruokonen A, Tichet J, Marre M, Weill J, Heude B, Tauber M, Lemaire K, Schuit F, Elliott P, Jorgensen T, Charpentier G, Hadjadj S, Cauchi S, Vaxillaire M, Sladek R, Visvikis-Siest S, Balkau B, Levy-Marchal C, Pattou F, Meyre D, Blakemore AI, Jarvelin MR, Walley AJ, Hansen T, Dina C, Pedersen O, Froguel P. A variant near MTNR1B is associated with increased fasting plasma glucose levels and type 2 diabetes risk. Nat Genet. 2009;41(1):89-94.

30. Bonnefond A, Clement N, Fawcett K, Yengo L, Vaillant E, Guillaume JL, Dechaume A, Payne F, Roussel R, Czernichow S, Hercberg S, Hadjadj S, Balkau B, Marre M, Lantieri O, Langenberg C, Bouatia-Naji N, Meta-Analysis of G, Insulin-Related Traits C, Charpentier G, Vaxillaire M, Rocheleau G, Wareham NJ, Sladek R, McCarthy MI, Dina C, Barroso I, Jockers R, Froguel P. Rare MTNR1B variants impairing melatonin receptor 1B function contribute to type 2 diabetes. Nature genetics. 2012;44(3):297-301.

31. Kozirog M, Poliwczak AR, Duchnowicz P, Koter-Michalak M, Sikora J, Broncel M. Melatonin treatment improves blood pressure, lipid profile, and parameters of oxidative stress in patients with metabolic syndrome. J Pineal Res. 2011;50(3):261-266.

32. Brouwer A, van Raalte DH, Diamant M, Rutters F, van Someren EJ, Snoek FJ, Beekman AT, Bremmer MA. Light therapy for better mood and insulin sensitivity in patients with major depression and type 2 diabetes: a randomised, double-blind, parallel-arm trial. BMC Psychiatry. 2015;15:169.

Table 1: Characteristics of the study populations

\begin{tabular}{|l|c|c|}
\hline & OBB $(\mathrm{N}=4,327)$ & NEO $(\mathrm{N}=5,899)$ \\
\hline Age in years, mean (SD) & $41.4(5.9)$ & $55.6(6.0)$ \\
\hline Men, $\%$ & 43.2 & 42.8 \\
\hline Body mass index in kg/m ${ }^{2}$ mean (SD) & $25.9(4.7)$ & $26.1(4.3)$ \\
\hline Percentage body fat, mean (SD) & $42.1(12.0)$ & $31.5(8.6)$ \\
\hline Glucose in mmol/L, mean (SD) & $5.2(0.5)$ & $5.3(0.5)$ \\
\hline Insulin in mIU/L, median (IQR) & $11.5(8.7,15.2)$ & $7.5(5.1,11.3)$ \\
\hline HOMA-IR, median (IQR) & $2.6(1.9,3.6)$ & $1.8(1.2,2.7)$ \\
\hline HOMA-B, median (IQR) & $106(89,126)$ & $87(61,126)$ \\
\hline LDL-cholesterol in mmol/L, mean (SD) & $3.2(1.2)$ & $3.6(1.0)$ \\
\hline
\end{tabular}




\begin{tabular}{|l|c|c|}
\hline HDL-cholesterol in mmol/L, mean (SD) & $1.4(0.4)$ & $1.6(0.5)$ \\
\hline Triglycerides in mmol/L, median (IQR) & $0.9(0.7,1.3)$ & $1.0(0.7 ; 1.5)$ \\
\hline Use of lipid-lowering medication, $\%$ & 0.7 & 24.2 \\
\hline Winter, $\%$ & 24.5 & 26.5 \\
\hline Spring, $\%$ & 25.0 & 24.3 \\
\hline Summer, $\%$ & 23.1 & 25.3 \\
\hline Autumn, $\%$ & 27.4 & $10.5(5.6)$ \\
\hline Outdoor temperature $\left(7\right.$ days) in ${ }^{\circ} \mathrm{C}$, mean $(\mathrm{SD})$ & $10.6(5.2)$ & $5.0(2.7,7.0)$ \\
\hline Outdoor bright sunlight in hours $(7$ days), median (IQR) & $3.8(2.4,5.9)$ & \\
\hline
\end{tabular}

Results of the NEO study population are weighted towards the body mass distribution of the general population. Abbreviations: HOMA-B, homeostatic model assessment for beta cell function; HOMA-IR, homeostatic model assessment for insulin resistance; IQR, interquartile range; NEO, Netherlands Epidemiology of Obesity; N, number of participants; OBB, Oxford Biobank; SD, standard deviation.

Table 2: Association between outdoor temperature and measures of glucose and lipid metabolism in the OBB and NEO study populations

\begin{tabular}{|c|c|c|c|}
\hline & Model 1 & Model 2 & Model 3 \\
\hline \multicolumn{4}{|l|}{ Glucose metabolism } \\
\hline Glucose, $\%$, OBB & $-0.06(-0.29,0.17)$ & $-0.51(-0.92,-0.09)$ & $-0.38(-0.85,0.09)$ \\
\hline Glucose, $\%$, NEO & $0.22(-0.09,0.52)$ & $0.19(-0.36,0.73)$ & $0.23(-0.33,0.78)$ \\
\hline Insulin, $\%, \mathrm{OBB}$ & $-1.10(-2.44,0.22)$ & $-2.22(-4.67,0.17)$ & $-0.79(-3.50,1.84)$ \\
\hline Insulin, $\%, \mathrm{NEO}$ & $-0.37(-2.21,1.44)$ & $-0.94(-3.85,1.95)$ & $-0.69(-3.70,2.41)$ \\
\hline HOMA-IR, $\%$, OBB & $-1.42(-2.86,-0.01)$ & $-3.11(-5.74,-0.54)$ & $-1.47(-4.38,1.36)$ \\
\hline HOMA-IR, \%, NEO & $-0.15(-2.08,1.82)$ & $-0.75(-3.75,2.34)$ & $-0.46(-3.81,2.76)$ \\
\hline HOMA-B, $\%$, OBВ & $-0.65(-1.48,0.18)$ & $-0.53(-2.04,0.96)$ & $0.19(-1.49,1.83)$ \\
\hline HOMA-B, $\%$, NEO & $-0.85(-2.56,0.82)$ & $-1.47(-4.55,1.52)$ & $-1.25(-4.49,1.88)$ \\
\hline \multicolumn{4}{|l|}{ Lipid metabolism } \\
\hline LDL-cholesterol, \%, OBB & $-0.20(-0.91,0.50)$ & $-0.33(-1.61,0.93)$ & $-0.08(-1.49,1.32)$ \\
\hline LDL-cholesterol, \%, NEO & $0.00(-0.86,0.84)$ & $1.26(-0.26,2.76)$ & $1.46(-0.14,3.04)$ \\
\hline HDL-cholesterol, \%, OBB & $-0.19(-0.92,0.53)$ & $-0.59(-1.91,0.71)$ & $-0.56(-2.04,0.90)$ \\
\hline HDL-cholesterol, \%, NEO & $-0.76(-1.55,0.03)$ & $-0.81(-2.20,0.56)$ & $-0.83(-2.28,0.60)$ \\
\hline Triglycerides, $\%, \mathrm{OBB}$ & $0.08(-1.21,1.35)$ & $-2.02(-4.40,0.31)$ & $-0.31(-2.93,2.25)$ \\
\hline Triglycerides, $\%$, NEO & $-0.37(-1.97,1.21)$ & $-0.25(-3.17,2.59)$ & $0.38(-2.58,3.27)$ \\
\hline
\end{tabular}

Presented associations are from the OBB $(\mathrm{N}=4,327)$ and NEO study $(\mathrm{N}=5,899)$ populations. Results from the Netherlands Epidemiology of Obesity Study are weighted toward to body mass index distribution of the general population. Associations are presented as the percentage difference in the outcome measure per 5 degrees Celsius increased outdoor temperature during the 7 days before the center visit (95\% confidence interval). Model 1, analyses adjusted for age, sex and percentage of body fat. Model 2, analyses adjusted for age, sex, percentage of body fat, and season. Model 3, analyses adjusted for age, sex, percentage body fat, season, outdoor bright sunlight. Abbreviations: HDL, high-density lipoprotein; HOMA-B, homeostatic model assessment for beta cell function; HOMA-IR, homeostatic model assessment for insulin resistance; LDL, low-density lipoprotein, NEO, Netherlands Epidemiology of Obesity; OBB, Oxford Biobank. 
Table 3: Association between outdoor bright sunlight and measures of glucose and lipid metabolism in the OBB and NEO study populations

\begin{tabular}{|l|c|c|c|}
\hline & Model 1 & Model 2 & Model 3 \\
\hline Glucose metabolism & & & $-0.03(-0.17,0.11)$ \\
\hline Glucose, \%, OBB & $-0.05(-0.15,0.05)$ & $-0.08(-0.21,0.04)$ & $-0.05(-0.22,0.12)$ \\
\hline Glucose, \%, NEO & $-0.02(-0.14,0.10)$ & $-0.04(-0.20,0.13)$ & $-1.27(-2.09,-0.47)$ \\
\hline Insulin, \%, OBB & $-0.92(-1.48,-0.36)$ & $-1.37(-2.09,-0.65)$ & $-0.36(-1.38,0.65)$ \\
\hline Insulin, \%, NEO & $0.21(-0.47,0.88)$ & $-0.40(-1.37,0.55)$ & $-1.36(-2.23,-0.50)$ \\
\hline HOMA-IR, \%, OBB & $-1.05(-1.65,-0.45)$ & $-1.54(-2.32,-0.77)$ & $-0.41(-1.50,0.67)$ \\
\hline HOMA-IR, \%, NEO & $0.19(-0.53,0.90)$ & $-0.44(-1.47,0.58)$ & $-0.80(-1.31,-0.30)$ \\
\hline HOMA-B, \%, OBB & $-0.54(-0.89,-0.19)$ & $-0.78(-1.23,-0.33)$ & $-0.31(-1.26,0.64)$ \\
\hline HOMA-B, \%, NEO & $0.27(-0.40,0.93)$ & $-0.39(-1.30,0.52)$ & $-0.16(-0.58,0.27)$ \\
\hline Lipid metabolism & & & $-0.28(-0.76,0.19)$ \\
\hline LDL-cholesterol, \%, OBB & $-0.36(-0.66,-0.07)$ & $-0.19(-0.57,0.19)$ & $-0.01(-0.45,0.43)$ \\
\hline LDL-cholesterol, \%, NEO & $-0.09(-0.44,0.25)$ & $-0.19(-0.64,0.26)$ & $0.02(-0.39,0.44)$ \\
\hline HDL-cholesterol, \%, OBB & $-0.25(-0.56,0.06)$ & $0.00(-0.39,0.39)$ & $-1.28(-2.07,-0.50)$ \\
\hline HDL-cholesterol, \%, NEO & $-0.10(-0.41,0.21)$ & $-0.03(-0.43,0.37)$ & $-0.91(-1.80,-0.03)$ \\
\hline Triglycerides, \%, OBB & $-0.57(-1.12,-0.03)$ & $-1.36(-2.07,-0.66)$ & $-0.89(-1.75,-0.03)$ \\
\hline Triglycerides, \%, NEO & $-0.97(-1.62,-0.32)$ & & \\
\hline Presented assOciatins & & & \\
\hline
\end{tabular}

Presented associations are from the OBB $(\mathrm{N}=4,327)$ and the NEO study $(\mathrm{N}=5,899)$ populations. Results from the Netherlands Epidemiology of Obesity Study are weighted toward to body mass index distribution of the general population. Associations are presented as the percentage difference in the outcome measure per hour increase in bright sunlight during the 7 days before the center visit (95\% confidence interval). Model 1, analyses adjusted for age, sex and percentage of body fat. Model 2, analyses adjusted for age, sex, percentage body fat, and season. Model 3, analyses adjusted for age, sex, percentage of body fat, season, outdoor temperature. Abbreviations: HDL, high-density lipoprotein; HOMA-B, homeostatic model assessment for beta cell function; HOMA-IR, homeostatic model assessment for insulin resistance; LDL, low-density lipoprotein; NEO, Netherlands Epidemiology of Obesity; OBB, Oxford Biobank. 